\title{
Evaluating the Road Safety Effects of a Fuel Cost Increase Measure by means of Zonal Crash Prediction Modeling
}

\author{
Ali Pirdavani ${ }^{1}$ \\ Transportation Research Institute (IMOB) \\ Hasselt University \\ Wetenschapspark 5 bus 6 \\ B-3590 Diepenbeek, Belgium \\ ali.pirdavani@uhasselt.be \\ Tel: +32(0)11269139 \\ Fax: +32(0)11269199 \\ Tom Brijs \\ Transportation Research Institute (IMOB) \\ Hasselt University \\ Wetenschapspark 5 bus 6 \\ B-3590 Diepenbeek, Belgium \\ tom.brijs@uhasselt.be \\ Tom Bellemans \\ Transportation Research Institute (IMOB) \\ Hasselt University \\ Wetenschapspark 5 bus 6 \\ B-3590 Diepenbeek, Belgium \\ tom.bellemans@uhasselt.be \\ Bruno Kochan \\ Transportation Research Institute (IMOB) \\ Hasselt University \\ Wetenschapspark 5 bus 6 \\ B-3590 Diepenbeek, Belgium \\ bruno.kochan@uhasselt.be \\ Geert Wets \\ Transportation Research Institute (IMOB) \\ Hasselt University \\ Wetenschapspark 5 bus 6 \\ B-3590 Diepenbeek, Belgium \\ Geert.wets@uhasselt.be
}

\footnotetext{
${ }^{1}$ Corresponding author
} 


\begin{abstract}
Travel Demand Management (TDM) consists of a variety of policy measures that affect the transportation system's effectiveness by changing travel behavior. The primary objective to implement such TDM strategies is not to improve traffic safety, although their impact on traffic safety should not be neglected. The main purpose of this study is to evaluate the traffic safety impact of conducting a fuel-cost increase scenario (i.e. increasing the fuel price by $20 \%$ ) in Flanders, Belgium. Since TDM strategies are usually conducted at an aggregate level, Crash Prediction Models (CPMs) should also be developed at a geographically aggregated level. Therefore Zonal Crash Prediction Models (ZCPMs) are considered to present the association between observed crashes in each zone and a set of predictor variables. To this end, an activitybased transportation model framework is applied to produce exposure metrics which will be used in prediction models. This allows us to conduct a more detailed and reliable assessment while TDM strategies are inherently modeled in the activity-based models unlike traditional models in which the impact of TDM strategies are assumed. The crash data used in this study consist of fatal and injury crashes observed between 2004 and 2007. The network and socio-demographic variables are also collected from other sources. In this study, different ZCPMs are developed to predict the Number of Injury Crashes (NOCs) (disaggregated by different severity levels and crash types) for both the null and the fuel-cost increase scenario. The results show a considerable traffic safety benefit of conducting the fuel-cost increase scenario apart from its impact on the reduction of the total Vehicle Kilometers Travelled (VKT). A 20\% increase in fuel price is predicted to reduce the annual VKT by 5.02 billion $(11.57 \%$ of the total annual VKT in Flanders), which causes the total NOCs to decline by $2.83 \%$.
\end{abstract}

Keywords: Crash prediction models; Traffic analysis zones; Transportation planning; Travel demand management; Safety planning; Fuel-cost increase scenario; Activity-based models. 


\section{Introduction}

It is beneficial to know the consequences of TDM (Travel Demand Management) strategies e.g. on traffic safety, which is considered to be an external side effect. Road crashes are known as one of the negative impacts of growing travel demand. For many years, researchers have attempted to investigate this impact by predicting the NOCs based on patterns they learned from crashes that occurred in the past. Traditionally, this reactive approach consists of different phases such as identification, diagnosis and improvement of dangerous locations, so-called hot spots. From an ethical point of view, this reactive approach is not acceptable because it requires several years of crashes to occur in order to identify and treat safety problems. Providing a more proactive approach, capable of evaluating road safety at the planning-level is therefore essential. In the last few years researchers and practitioners have increasingly applied this proactive approach. Dealing with traffic safety at the planning level requires the ability to integrate a crash predicting context into TDM strategies. TDM consists of several policies and strategies which aim to overcome transportation problems in different ways, e.g. changing travel behavior, making transportation systems more efficient or reducing travel demand. In general, TDM strategies are implemented to improve transportation systems' efficiency. However their potential traffic safety impact should not be ignored. TDM strategies improve transport system efficiency by means of mode shift (e.g. using public transportation instead of cars, biking for short distance trips or carpooling), travel time shift (e.g. avoiding traffic peak-hours by leaving home/the work place earlier or later), or travel demand reduction (e.g. teleworking) (VTPI, 2011). TDM strategies are usually performed and evaluated at geographically aggregated levels rather than merely the level of individual intersections or road sections. Therefore the impact of adopting a TDM strategy on transportation or traffic safety should also be evaluated at a level higher than merely the local consequences.

The application of CPMs (Crash Prediction Models) at a geographically aggregated level such as a Traffic Analysis Zone (TAZ) leads to a ZCPM (Zonal Crash Prediction Model). Until now, ZCPMs have hardly ever been incorporated in TDM strategies. The main goal of this study is therefore to integrate ZCPMs with a fuel-cost increase scenario to evaluate the traffic safety effects of conducting such a TDM strategy by means of a simulation-based analysis of the impact of fuel price on the travel demand in Flanders, Belgium. This way, the behavioral impact of the TDM scenario in terms of traffic demand is incorporated in the analysis. By assigning traffic demand to the road network and using this information at zonal level, the impact of responses to TDM, such as changes in trip planning, route choice and modal choice are incorporated in the analysis. This study is an assessment exercise which illustrates the impact of a $20 \%$ increase in fuel-related costs on traffic safety. It is essential to note that this $20 \%$ increase in fuel-related costs (expressed in $€$ per kilometer) is not an optimized value and is the result of a change in fuel price (expressed in $€$ per liter) on the one hand and fuel economy (liter per kilometer) on the other hand.

To account for severity of crashes, different ZCPMs are developed at different severity levels; i.e. "fatal + severe injury" and "slight injury" crashes. Additionally, in order to represent the mode shift effects, crashes are disaggregated into two different types namely "Car-Car" and "Car-Slowmode" crashes ("Slowmode" comprises pedestrians and cyclists). Accordingly, four different ZCPMs are developed to explain the traffic safety impacts of the fuel-related cost increase at different crash type/severity levels. 
It is necessary to indicate that the FEATHERS model (Bellemans et al. 2010) models the transportation demand of a population who is aware of the state of the transportation network. Hence, the assumed travel times during the activity-travel planning phase are in correspondence with the travel times obtained after assigning the total traffic demand to the road network (this is achieved through iteration). This means that the model is a steady state model and that no transients are modeled. Moreover, the model is a short term model in the sense that it does not assume a shift in the composition of the vehicle fleet as a result of the change in fuel cost. This assumption is justified by the slower time scale of vehicle fleet composition (in the order of several months to even years). Also changes in the location of businesses and/or the location choice for living (i.e. land use characteristics) occur at a far slower time scale than the adaptation of travel behavior to changing fuel cost triggers.

The structure of this paper is as follows. Initially, we will review the literature. Then the activity-based model which is used in this study will be briefly introduced. In the next sections the data preparation and the fuel-cost increase scenario evaluation process will be demonstrated. Finally, the results of this evaluation will be shown followed by the final conclusions and discussions.

\section{Literature review}

TDM strategies have hardly ever been implemented to improve traffic safety. Their main objectives are usually the reduction of congestion and emission, as well as travel cost and energy by means of reducing travel demand and consequently vehicle distance travelled. Nevertheless, apart from improving the efficiency of the transportation system and economic/environmental benefits, identifying the traffic safety impacts of a TDM strategy, can strengthen the implementation of such a strategy. It is a well-known fact in literature that road crashes are tightly linked to traffic exposure. Therefore, strategies that reduce travel demand or distance travelled, or cause a modal shift to a safer mode (e.g. from car to public transportation) tend to reduce the NOCs (Litman 2006; Litman 2011).

Lovegrove and Litman (2008) applied community-based, macro-level CPMs to calculate the road safety impacts of three mobility management strategies: smart growth, congestion pricing and improving transport options. They assumed the effect of implementing these strategies on different explanatory variables of the CPMs (e.g. they found that the smart growth strategy will decrease vehicle kilometers traveled (VKT) by 15\%). Based on these assumptions the expected NOCs have been calculated for each TDM strategy. The results indicated that mobility management strategies can significantly improve traffic safety.

Fuel-related costs are a major component of each motor vehicle's operating expenses. By increasing the fuel price as a TDM strategy, people tend to travel less by car, and instead use public transportation, carpool, or shift towards slow modes (biking and walking) etc. Thus, traffic crashes are expected to decrease as a result of a reduction in the number of car kilometers traveled. Fuel-related costs have an impact on traffic safety through changes in travel demand. Grabowski and Morrisey (2004) reported a relatively stable number of fatal motor vehicle crashes despite new traffic safety laws and vehicle innovations over a period of time. Their explanation was that the price of gasoline declined, which resulted in more vehicle miles traveled and potentially more fatalities. Chi et al. (2010) also investigated the impact of gasoline price changes on different types of crashes at a more disaggregated level for different ages and genders. In their reactive approach, they developed models to predict traffic crashes based on 
explanatory variables like exposure, gasoline price, alcohol consumption, seat belt usage, etc. It was concluded that an increase in gasoline price both has a short-term and intermediate-term effect on reducing total traffic crashes. One of the longer-term effects of a fuel cost increase is the change of the fleet composition to more fuel-economic vehicles, which can partially compensate the increased fuel price by an increased fuel economy. In literature it is described (Goodwin et al. 2004; Litman 2010) that the fuel price elasticity of fuel consumption ranges from -0.25 to -0.6 , the elasticity of fuel efficiency ranges from 0.3 to 0.4 and the vehicle mileage elasticity ranges from -0.1 to -0.3 .

CPMs can be categorized in two different levels: the local level (road and intersection) and the regional level (e.g. TAZ). Usually local level CPMs aim to predict the safety benefits/detriment of infrastructural improvements. These models are not typically designed to evaluate traffic safety impacts of TDM strategies; thus, the application of CPMs at a higher aggregation level will be more practical (Tarko et al. 2008). The application of CPMs at TAZ level has been initially introduced by Levine et al (1995). In their study, a set of both socioeconomic and road network variables were chosen to predict the NOCs in TAZs. They developed a linear relationship between different explanatory variables and the NOCs.

Recently, the application of ZCPMs became more popular amongst researchers. Several researchers examined the association of a collection of network infrastructure variables, sociodemographic and socio-economic variables and weather conditions with the NOCs in TAZs (Aguero-Valverde and Jovanis 2006; Amoros et al. 2003; Huang et al. 2010; Noland and Oh 2004; Noland and Quddus 2004; Quddus 2008; Wier et al. 2009). It was found that the number of lanes, road length and road density were significantly correlated with the NOCs. As for the demographic variables, it was found that TAZs with a higher percentage of population under the poverty level and a higher percentage of population in the young and also elderly age groups have the potential of increasing crash risk. It was also found that the traffic safety situation is worse for TAZs with lower income and education levels and a higher unemployment rate compared to relatively affluent TAZs. In another study by Wier et al. (2009) it was shown that traffic volume, population size, the proportion of arterial streets without public transit, the proportion of population living in poverty, and the number of people aged 65+ as percentage of the total population, were significantly good predictors of crashes. Moreover, Noland and Quddus (2004) concluded that TAZs with high employment density had more traffic crashes, while in urbanized more densely populated TAZs fewer crashes were observed. De Guevara et al. (2004) developed planning-level ZCPMs for the city of Tucson, Arizona. They considered many socio-demographic and road network variables in their model construction. They concluded that predictors such as population density, the number of persons younger than 17 years old as a percentage of the total population, the number of employees, the intersection density, the percentage of miles of principal arterials, the percentage of miles of minor arterials and the percentage of miles of urban collectors are significant predictors for the NOCs.

Hadayeghi et al. (Hadayeghi 2009; Hadayeghi et al. 2003; Hadayeghi et al. 2006; Hadayeghi et al. 2007; Hadayeghi et al. 2010a, 2010b) have been working on ZCPMs for several years. In one of their first studies, it was shown that the number of accidents in a TAZ increases when the VKT, major and minor road length, total employed labor force, household population, and intersection density increase whereas it decreases with a higher posted speed and a higher level of congestion in the TAZ (Hadayeghi et al. 2003). Hadayeghi et al. (2006) investigated the temporal transferability of the ZCPMs by applying models constructed on 1996 data to predict the NOCs for each TAZ in 2001 for the City of Toronto. They concluded that the models are not 
transferable statistically but that VKT, socioeconomic and demographic parameters are significantly stable over time. In another research, twenty-three regression models were developed to examine the relationships between several types of transportation planning variables and collision frequency. Models were developed for each planning category individually and in combination with other categories. A comparison of the models' performance showed that the comprehensive models are performing statistically better than the individual models. The results showed the potential of planning-level safety models to provide decision support tools for planners to consider safety in the planning phase (Hadayeghi et al. 2007).

Hadayeghi et al (2010a) conducted the same research but this time they applied Geographically Weighted Poisson Regression (GWPR) instead of taking a Generalized Linear Modeling (GLM) approach. The major difference between these two types of models is that GWPR models allow the model coefficient estimates to vary spatially for each TAZ. This very important additional attribute of these models provides some extra information as it takes the spatial location of a crash into consideration.

Lovegrove and Sayed (2006) concluded that quantifying the relationship between the zonal characteristics such as exposure, network, socio-demographic and TDM variables and crashes at a zonal level provides a predictive tool to predict the NOCs in a TAZ. They used GLM techniques to develop ZCPMs for both urban and rural areas across the Greater Vancouver Regional District (GVRD). Their results show that increasing signal density, intersection density per unit area and per lane kilometers, arterial-local intersections in rural areas and total arterial road lane kilometers will lead to an increase in the NOCs. On the contrary, an increase in the number of three-leg intersections and local road lane kilometers will decrease the NOCs in a TAZ. Lovegrove and Sayed (2007) further developed a set of ZCPMs for a "black-spot" study in the GVRD. These sets of ZCPMs consist of an exposure variable (VKT) and other network, socio-demographic and TDM variables. The results of this study also confirmed that ZCPMs have the potential to complement traditional reactive road safety improvement programs.

Recently, some researchers constructed ZCPMs by associating the NOCs in a TAZ with trip production/attraction and other network characteristics. Abdel-Aty et al. (2011a) identified and prioritized important variables which can be associated with crashes per TAZ by means of the Classification and Regression Trees technique. It was shown that this methodology will be helpful in incorporating proactive safety measures for long-range transportation planning. They (Abdel-Aty et al. 2011b) also developed different ZCPMs for different crash severity levels and concluded that different sets of predictors should be considered based on the type or severity of crashes (e.g. total trip productions and attractions provide a better model fit for the total and peak hour crashes while severe crashes were best predicted by different trip-related variables). Naderan and Shahi (2010) investigated the possibility of associating travel demand in urban areas with crash frequencies in each TAZ. They developed a series of ZCPMs using the Number of Trips (NOTs) produced/attracted as predictors. They concluded that these models provide the basic tool for evaluating TDM scenarios in urban transportation planning in terms of traffic safety as the application of a specific TDM scenario may reduce trip productions of a specific motive. The drawback of considering only trips as an exposure variable is that the impact of trip time, trip length, route choice, intrazonal traffic and transit traffic on a TAZ will be neglected. The number of produced/attracted trips might be an acceptable indicator of how busy or active a TAZ is or how much people are exposed to dangerous situations, but it always leaves out the effects of through traffic which is just passing through a TAZ neither having their origin or destination in that TAZ. 
Although most of the above-mentioned studies were trying to demonstrate their potential as a predictive tool at planning level, so far not much attention has been paid to the application of these models to evaluate the effect of TDM's on traffic safety. There are very few attempts at estimating the road safety benefits of applying a specific TDM strategy. In a study conducted by Lovegrove and Litman (2008), they assumed the effect of implementing these strategies on different explanatory variables of the CPMs. Based on these assumptions the expected NOCs were calculated for each TDM strategy. For instance, it was concluded that a smart growth strategy of more compact and multi-modal land use development patterns may increase traffic safety by means of reducing crash frequency per capita by $20 \%$ and $29 \%$ for total and severe crashes respectively. An et al. (2011) found Vehicle Hours Travelled (VHT), the number of intersections and the number of households with low income levels to be correlated with the NOCs in TAZs. After running two add-capacity projects in the Pikes Peak region and applying the results in their developed ZCPMs for the do-nothing scenario and both project scenarios, total crashes for both projects were estimated to decrease respectively by $0.1 \%$ and $0.06 \%$ when compared with the do-nothing scenario.

According to literature, exposure is the most important predictor of crashes Therefore, having a more informative measure of exposure is expected to result in a better crash prediction. When a TDM scenario is performed, it basically changes the exposure compared with the null scenario. Thus, it is essential to predict the exposure metrics as accurately as possible. Activitybased models help with this as they are able to simulate the scenarios and in this case, they model the decision process of individuals with respect to the changes in fuel price. This is the key advantage of applying activity-based models rather than making educated guesses about the impact of fuel-related cost changes on travel demand in order to obtain exposure. In the next section the activity-based model is briefly introduced and its contribution to the fuel-cost increase scenario evaluation process is described.

\section{Impact of fuel-related cost on traffic demand}

Traditionally, travel was assumed to be the result of four subsequent decisions which were modeled separately, also referred to as four-step models. More recently, several researchers claimed that travel has an isolated role in these models and the reason why people undertake trips is neglected completely. This is why activity-based models have been taken into consideration. The main difference between four-step models and activity-based transportation models is that the latter try to predict interdependencies between several facets of activity profiles (Davidson et al. 2007). Hence, activity-based models are designed to keep the linkages between the travel decisions of individual members of a single household. Interactions among family members such as the use of household vehicles, sharing household responsibilities or performing joint activities, often affect and in many cases largely determine people's travel. Four-step models that ignore such linkages, misstate people's responses to TDM strategies. It is shown that activity-based models are capable of treating TDM strategies and policy issues whereas four-step models become ineffective (Vovsha and Bradley 2006).

\subsection{FEATHERS framework}

The FEATHERS (Forecasting Evolutionary Activity-Travel of Households and their Environmental RepercussionS) framework (Janssens et al. 2007) was developed in order to facilitate the development of activity-based models for transportation demand in Flanders, 
Belgium. The scheduling engine that is currently implemented in the FEATHERS framework is based on the scheduling engine that is present in the Albatross system (Arentze and Timmermans 2004). Currently, the framework is fully operational at the level of Flanders. The real-life representation of Flanders is embedded in an agent-based simulation model which consists of over six million agents, each agent representing one member of the Flemish population. A sequence of 26 decision trees, derived by means of the CHisquared Automatic Interaction Detector (CHAID) algorithm, is used in the scheduling process and decisions are based on a number of attributes of the individual (e.g. age, gender), of the household (e.g. number of cars) and of the geographical zone (e.g. population density, number of shops). For each agent with its specific attributes, the model simulates whether an activity (e.g. shopping, working, leisure activity ...) is going to be carried out or not. Subsequently, amongst others, the location, transport mode and duration of the activity are determined, taking into account the attributes of the individual (Kochan et al. 2008). Traffic demand is subsequently assigned to the road network in such a way that an equilibrium is established between transportation demand and supply (Bellemans et al. 2010), which results in a time-dependent traffic state on the road network. In order to run, calibrate and validate the activity-based model, three major types of data are required (Kochan et al. 2011); data describing the environment (e.g. population density, level of service of the transportation networks), a synthetic population which is simulated and activity-travel data originating from a representative sample of the population from which the human behavior is derived. Screen line counts on the road network can also be used to validate the model.

\subsection{Implementation of fuel-cost increase scenario in FEATHERS}

An important asset of activity-based models in this context is their integrated approach towards activities and travel. Due to this approach, it can be taken into account that certain trips, which are linked to activities that are not so flexible (such as e.g. work activities) are less likely to be altered under changing traffic system conditions than others (such as e.g. leisure activities). In addition, activity-based models are not only able to predict a change in the demand for travel, but they also predict shifts between different modes of transport and the reallocation of activities due to the imposed measures. Providing a structured approach to agent-based modeling of activities and travel for individuals, the FEATHERS framework is able to account for TDM strategies. For instance, when applying a fuel cost increase scenario, FEATHERS can predict the impact on the NOTs, modal shift and changes in trip time and length.

However, price changes can have an impact on different facets of travel, affecting the NOTs people undertake, their destination, route, mode, travel time, type of vehicle (including size, fuel efficiency and fuel type) and parking location and duration. Therefore, in order to predict the impact of price changes like fuel price, the scheduling engine has to be structured to account for those changes. In this scheduling engine, price and cost parameters are incorporated in the decision trees related to activity selection, timing, trip-chaining, location and mode choices. The extended decision trees or Parametric Action Decision Trees combine conventional decision trees and parametric action assignment rules yielding a model that is sensitive for travelcosts scenarios (Arentze and Timmermans 2005).

In this study, fuel-related cost is assumed to increase by $20 \%$ as a result of an increase in fuel price. We consider the short term effect and can as such neglect the rebound effect caused by a changing fuel economy of the fleet (i.e. the fuel economy is considered to remain constant, which results in the reduction of fuel consumption to be equal to the reduction of the VKT). One 
might question how the impact of this global fuel price increase will be sensible at zonal level. As mentioned earlier, each zone has its own characteristics: the level of income, availability of public transportation, major activity types, etc. are different from zone to zone. These differences result in different travel behavior and more specifically different mode choice by the inhabitants of each zone. Therefore, despite the fact that the fuel price increase is applied globally, its impact is dissimilar from zone to zone.

\section{Data preparation}

The study area in this research is the Dutch-speaking region in northern Belgium, Flanders. Flanders has over 6 million inhabitants, or about $60 \%$ of the population of Belgium. As already mentioned before, an activity-based model within the FEATHERS framework is applied on the Flemish population to derive the in-depth information of Flemish peoples' travel behavior and travel demand for a null-scenario (current situation) and some TDM scenarios like increasing fuel price, teleworking, etc. FEATHERS produces traffic demand by means of OriginDestination (OD) matrices. These OD matrices include the number of trips for each traffic mode at different disaggregation levels (i.e. age, gender, day of the week, time of day and motive). This traffic demand is then assigned to the road network to obtain detailed exposure metrics at network level. To carry out the assignment of car trips to the road network, the user equilibrium method was selected. The fundamental nature of equilibrium assignment is that travelers will strive to find the shortest path (e.g. minimum travel time) from origin to destination, and network equilibrium occurs when no traveler can decrease travel effort by shifting to a new path. This is an optimal condition, in which no user will gain from changing travel paths once the system is in equilibrium. Exposure metrics are then geographically aggregated to the TAZ level. This has been carried out at zonal level, comprising 2,200 TAZs in Flanders. The average size of TAZs is 6.09 square kilometers with standard deviation of 4.78 square kilometers. In addition, for each TAZ a set of variables including socio-demographic and road network variables were derived to construct ZCPMs. The crash data used in this study consist of a geo-coded set of fatal and injury crashes that occurred during the period 2004 to 2007 which was provided by the Flemish Ministry of Mobility and Public Works. Table 1 shows a list of selected variables, together with their definition and descriptive statistics, which have been used in developing the ZCPMs presented in this paper. 
TABLE 1 Selected Variables to Develop ZCPMs

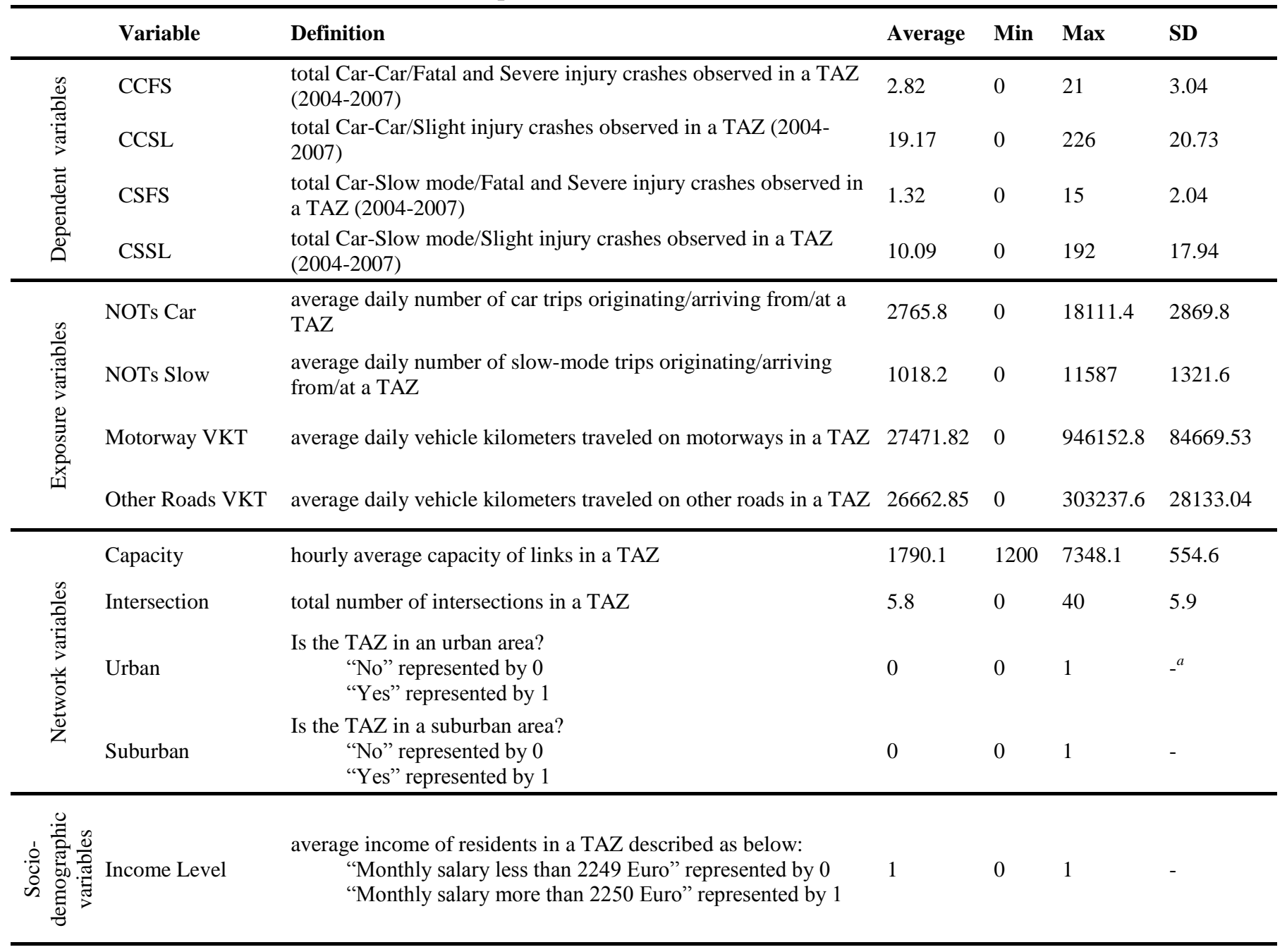

a: Data not applicable.

\section{Fuel-cost increase scenario evaluation}

\subsection{Model development}

Crash data consist of non-negative integers, so using ordinary least-squares regression which serves continuous dependent variables (e.g. time) is not an option (Lord and Mannering 2010). Given the observed overdispersion in the crash data in this study, it was chosen to model the data using the Negative Binomial (NB) model which enables the modeling of overdispersed data. The NB model is the most commonly used model in crash data modeling (Lord and Mannering 2010). In this study, different Negative Binomial (NB) ZCPMs were constructed within the Generalized Linear Modeling (GLM) framework, using the explanatory variables listed in Table 1. The models can be categorized into three different groups based on the type of exposure metric that was utilized, i.e. 1) flow-based models, 2) trip-based models and 3) models based on a combination of the two. Flow-based models were constructed by associating the NOCs in each TAZ with VHT or VKT, as the exposure variables, and the road network and socio-demographic variables listed in Table 1. Trip-based models use the same road network and 
socio-demographic variables but NOTs as the exposure variable. In the third type of model, both flow and trip-based variables are included simultaneously as metrics of exposure. Coefficients were estimated using a forward selection procedure where one of the exposure variables is taken as the starting point, subsequently selecting additional candidate variables. The analysis results revealed that the combination of exposure variables provides a better model fit; i.e. the models which simultaneously have both NOTs and VHT/VKT as the exposure variables overperform the flow-based or trip-based models (Pirdavani et al. 2012). Based on different statistical tests of goodness-of-fit, the final ZCPMs have been chosen. Table 2 provides the final models' estimates of the different explanatory variables. These are the models by which the fuel-cost increase scenario is being evaluated.

TABLE 2 Model Estimates for the Final Chosen ZCPMs

\begin{tabular}{lllll}
\hline & $\begin{array}{l}\text { Model \#1 } \\
\text { (CCFS) }\end{array}$ & $\begin{array}{l}\text { Model \#2 } \\
(\text { CCSL) }\end{array}$ & $\begin{array}{l}\text { Model \#3 } \\
(\text { CSFS })\end{array}$ & $\begin{array}{l}\text { Model \#4 } \\
\text { (CS/SL) }\end{array}$ \\
\hline Coefficients & Estimates & Estimates & Estimates & Estimates \\
\hline (Intercept) & $-4.356 \mathrm{e}+00$ & $-4.539 \mathrm{e}+00$ & $-7.357 \mathrm{e}+00$ & $-6.802 \mathrm{e}+00$ \\
$\log ($ NOTs Car) & $1.162 \mathrm{e}-01$ & $3.990 \mathrm{e}-01$ & - & - \\
$\log ($ NOTs Slow) & ${ }^{\mathrm{a}}$ & - & $7.445 \mathrm{e}-01$ & $9.005 \mathrm{e}-01$ \\
$\log ($ Motorway VKT) & $1.464 \mathrm{e}-02$ & $1.784 \mathrm{e}-02$ & $-2.528 \mathrm{e}-02$ & $-1.267 \mathrm{e}-02$ \\
$\log ($ Other Roads VKT) & $3.693 \mathrm{e}-01$ & $3.379 \mathrm{e}-01$ & $2.144 \mathrm{e}-01$ & $2.280 \mathrm{e}-01$ \\
Income Level & - & $-1.116 \mathrm{e}-01$ & $-1.643 \mathrm{e}-01$ & $-1.268 \mathrm{e}-01$ \\
Capacity & $3.342 \mathrm{e}-04$ & $4.140 \mathrm{e}-04$ & $2.018 \mathrm{e}-04$ & $1.716 \mathrm{e}-04$ \\
Intersection & $3.128 \mathrm{e}-02$ & $3.195 \mathrm{e}-02$ & $1.959 \mathrm{e}-02$ & $1.397 \mathrm{e}-02$ \\
Urban & $-4.455 \mathrm{e}-01$ & - & - & $5.678 \mathrm{e}-01$ \\
Suburban & $-2.194 \mathrm{e}-02$ & - & - & $2.673 \mathrm{e}-01$ \\
PCC & 0.615 & 0.831 & 0.721 & 0.855 \\
\hline
\end{tabular}

a: Data not applicable.

b: Pearson Correlation Coefficient (PCC) between observed and predicted crash values

For all models, most of the exposure variables were positively associated with the NOCs in each TAZ. As the NOTs and VKT increase, the total NOCs also tends to increase. Many studies found similar associations between VKT (e.g. Hadayeghi et al. 2010a, 2010b, Lovegrove 2005) or NOTs (e.g. Abdel-Aty et al. 2011a, 2011b, Naderan and Shahi 2010) and NOCs per TAZ. The only exception was observed where VKT on motorways was negatively associated with "Car-Slowmode" crashes. This is in line with the fact that the more VKT carried out on motorways (where there is no "Slowmode" traffic) instead of other roads, less vulnerable road users are exposed to unsafe situations.

A positive correlation of the number of intersections with the NOCs per TAZ is observed for all models. This positive relationship has also been reported in other studies (e.g. Hadayeghi et al. 2003, de Guevara et al.2004, An et al. 2011). In general, intersections have a higher risk of experiencing conflicts compared to road links because of their natural design, therefore there are more crashes expected to occur in TAZs that have a higher number of intersections. As can be observed in Table 2, all of the constructed models showed a negative association with "Income 
Level". This is similar to other studies' findings that poverty has a positive relationship with the number of crashes that occurred in a TAZ (e.g. Quddus 2008, Wier et al. 2009, Huang et al. 2010).

The degree of urbanization is categorized into three different levels and therefore represented by two dummy variables; "Urban" and "Suburban". When "Urban" and "Suburban" metrics in a TAZ are both 0 , then this TAZ is located in a rural area. In Model \#1, the coefficient estimate for the variables "Urban" and "Suburban" have negative signs. This is in line with our expectations that CCFS crashes are expected to occur more frequently in rural areas where cars are driven at higher speeds. On the contrary, the positive association between "Urban" and "Suburban" variables and CSSL crashes reveals that the model correctly predicts more crashes of this type in more urbanized TAZs.

\subsection{Traffic safety evaluation process}

OD matrices for the fuel cost increase scenario will be derived for scenario evaluation by running the activity-based transportation model. After assigning this demand to the road network, all required variables become available to set up the evaluation task. Now, the final ZCPMs are applied and crashes are predicted for each TAZ. The traffic safety evaluation can then be conducted by comparing the NOCs predicted by the final ZCPMs for the null and the fuel-cost increase scenario. Fig. 1 depicts the conceptual framework of the traffic safety evaluation process.

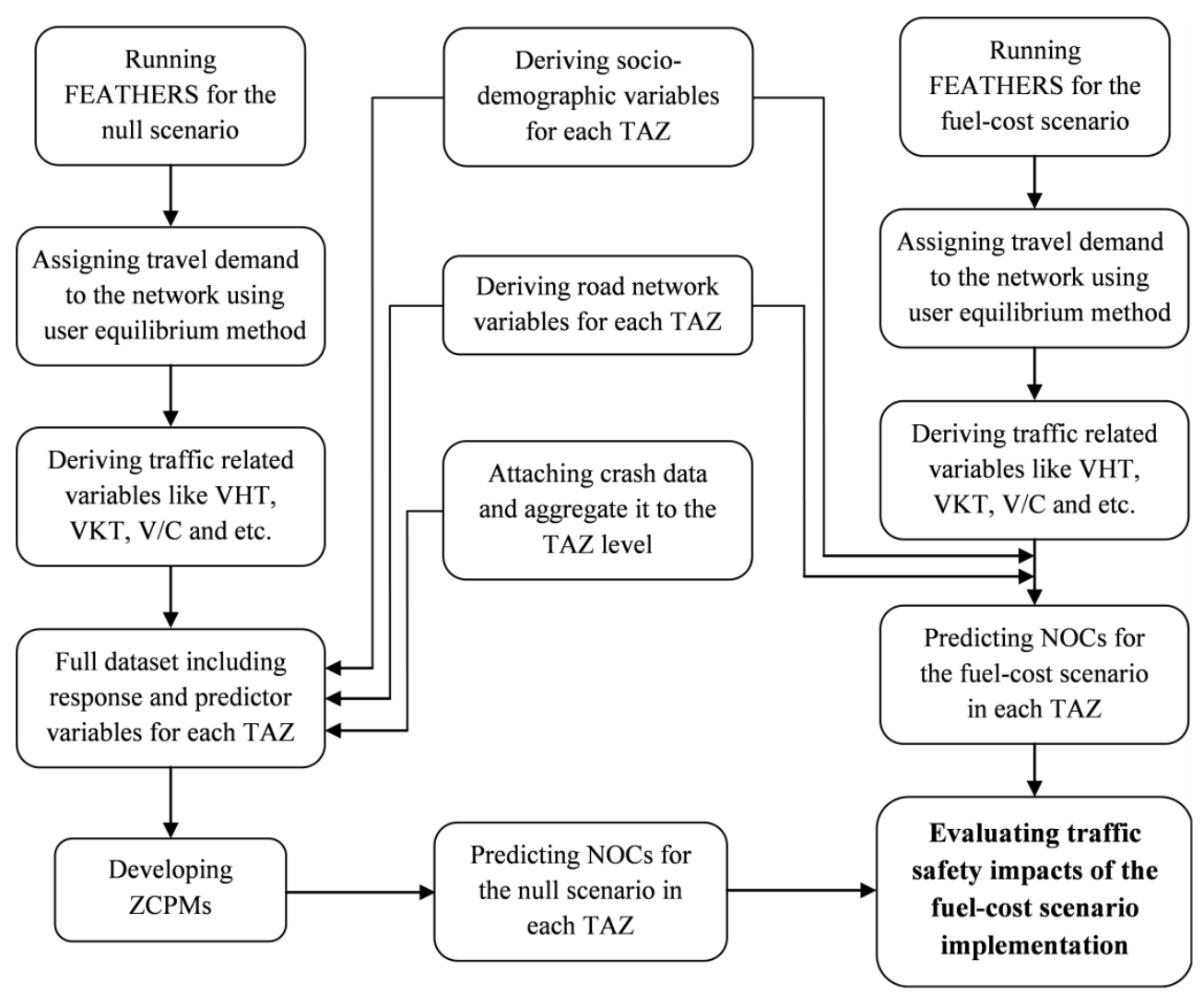

Fig. 1. Conceptual framework of the traffic safety evaluation process. 


\section{Results}

Before describing the traffic safety impact of the fuel-cost increase scenario, it would be beneficial to have a look at the changes made to the more traffic-related attributes playing a role in the whole chain. As described before, increasing fuel-related costs will affect and increase the total travel expenses of motor vehicle trips. As a result, people will start comparing the relative costs of travelling and may consider a shift to other available transportation modes. For instance, short-distance trips can be substituted by public transportation (e.g. bus or tram) or slow mode (i.e. biking or walking) or long-distance trips may shift towards public transportation (e.g. train) or be substituted by carpooling. Comparing OD matrices derived from the activity-based model for both the null and the fuel-cost increase scenario, will enable us to perceive any changes in NOTs for different modes and will also allow us to figure out if any mode shift will occur. The results of these comparisons revealed that the fuel-cost increase scenario reduces the average daily NOTs carried out by car $(-4.068 \%)$ and in contrast, leads to an increase in the average daily NOTs by other modes (Car Passenger by $+2.904 \%$, Public transportation by $+4.358 \%$ and Slow mode by $+2.863 \%$ ). In addition to these changes observed at the global level, it is also interesting to describe these changes at the TAZ level. It was observed that more urbanized areas experience a higher reduction of car trips as well as a higher increase of other mode trips when compared to less urbanized areas. On the contrary and in some particular TAZs like the ones nearby the Flemish borders, fewer mode shifts occurred. The reason might be that the crossborder public transportation offer is not a convenient option or no cross-border public transportation service is available in these TAZs. Thus, many travelers who are travelling across borders prefer to take their car since they cannot easily find a substitution mode.

Although the NOTs may represent an acceptable indication of exposure, it ignores the impact of transit traffic which is just passing through a TAZ. As already mentioned before, NOTs do not contain any information about trip time, trip length, route choice, intrazonal traffic and transit traffic. Therefore, investigating the impact of a fuel-cost increase scenario cannot be practically carried out by merely considering the changes in the NOTs starting or arriving in a TAZ. Thus, other exposure variables which can account for the impact of trip assignment should be taken into account. As a result, inclusion of the flow-related variables (e.g. VKT) in the prediction models is essential.

The analyses show that the average values of the VKT and VHT decrease after implementing the fuel-cost increase scenario. Not surprisingly, the reductions for motorways are higher than for other roads (i.e. reduction in motorway VHT by $16.85 \%$, other roads VHT by $10.34 \%$, motorway VKT by $13.31 \%$ and other roads VKT by $9.78 \%$ ). This can be explained by the fact that the majority of reduced long-distance trips are carried out on motorways. It also indicates that this scenario has a somewhat higher effect on long-distance trips compared to short-distance trips. It can also be noticed that the reduction in VHT is slightly higher than the reduction in VKT. A stronger decrease of travel time compared to travel distance can be explained by a decreased level of congestion on the roads. As the rebound effect of the vehicle fleet economy was not taken into account in this short-term analysis, we find that the reductions in VKT under the scenario are in line with the reductions in fuel consumption reported in literature (Goodwin et al. 2004; Litman 2010).

Predictably, in the fuel-cost increase scenario the total number of predicted "Car-Car" crashes decreases compared to the null scenario. This is due to reduced exposure as the main predictor of crashes. The results show that CCFS and CCSL crashes are predicted to decrease by 
248.4 and 2082.9 respectively for a period of 4 years $(-3.97 \%$ and $-4.77 \%)$. On the contrary, for "Car-Slowmode" crashes a slight increase is observed for both fatal-severe and slight injury crashes. Taken as a whole, CSFS and CSSL crashes are predicted to increase by 13.6 and 184.6 respectively for the same period of 4 years $(+0.46 \%$ and $+0.82 \%)$. Fig. 2 represents the violin plots of changes in NOCs after the fuel-cost increase scenario implementation. The violin plot is a synergistic combination of the box plot and the density trace (Hintze and Nelson 1998). These plots retain much of the information of box plots (except for the individual outliers), besides providing information about the distributional characteristics of the data. In these plots, the wider the violin, the more data points are associated to that value. Moreover, the white dots indicate the median; black boxes show the upper and lower quartile and the vertical black lines denote the upper and lower whiskers.

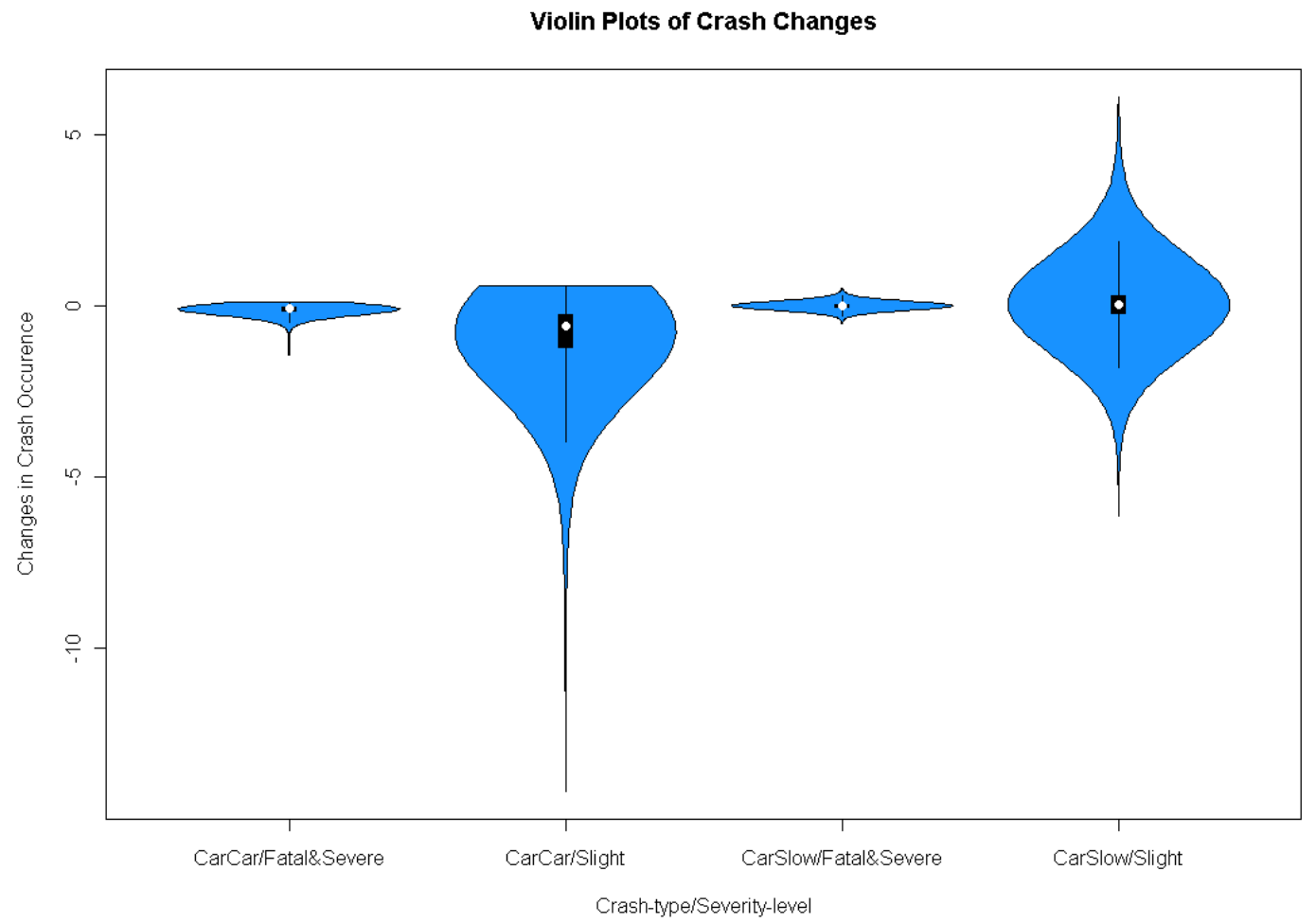

Fig. 2. Violin plots of changes in crash occurrence after the fuel-cost increase scenario implementation

In the development of "Car-Slowmode" models, both car and Slowmode-related exposure variables were used. Following the implementation of the fuel-cost increase scenario and as a result of mode shift, the number of car trips decreased whereas the number of Slowmode trips increased. However, these mode shifts are not always similar in all TAZs; i.e. more urbanized areas have a higher number of mode shifts; consequently more Slowmode-related crashes are predicted for these areas. An illustration of changes in the NOCs for all TAZs may present a better pattern on how different TAZs are affected by the scenario. In Fig. 3, the changes in the predicted NOCs are displayed for each TAZ. Fig. 3 reveals that the reductions in CCFS and CCSL crashes are greater for urban areas and generally smaller for TAZs close to the Flemish borders. As explained earlier, CSFS and CSSL crashes are predicted to increase in more 
urbanized areas; this is evident from the corresponding maps in Fig. 3 where concentrations of red dots stand for the major cities in Flanders.

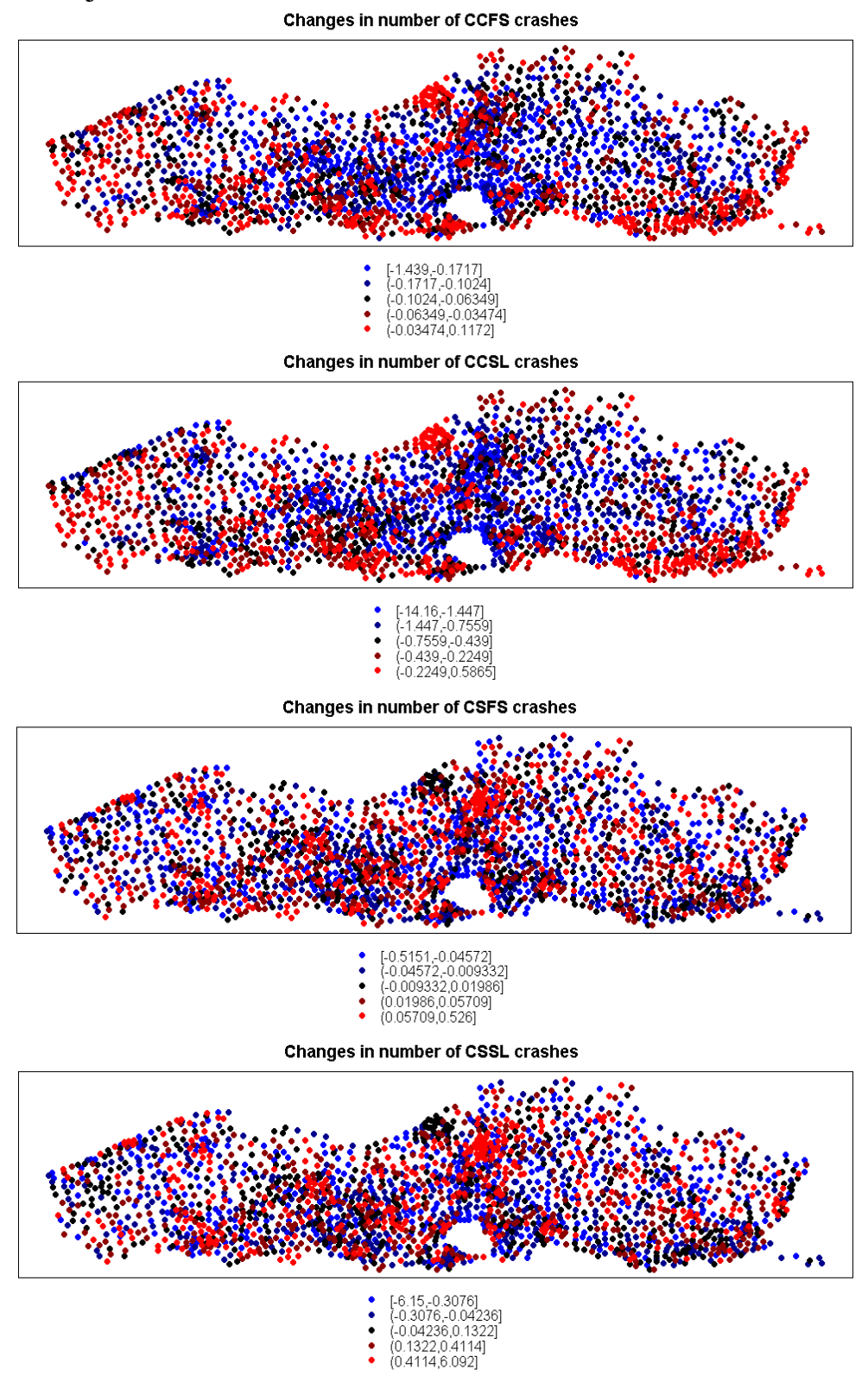

Fig.3. Changes in NOCs in each TAZ after the fuel-cost increase scenario implementation.

\section{Conclusions and discussion}

In this study, a zonal crash prediction modeling approach has been integrated into a fuelcost increase scenario to assess this TDM strategy's impact on traffic safety. This assessment deals with the relatively short-term impact of fuel price changes and it has been carried out by applying an activity-based travel demand model to derive the exposure metrics. Based on the results of the analyses, the following conclusions can be drawn: 
- Activity-based transportation models provide an adequate range of in-depth information about individuals' travel behavior. The advantage of these models is that the impact of applying a TDM strategy will be accounted for, for each individual, throughout a decision making process. Activity-based models provide more reliable information since, unlike traditional models, TDM strategies are inherently accounted for in these models.

- Unlike some prior studies where a reactive approach was generally used to evaluate the traffic safety impact of a TDM strategy, in this study a proactive methodology was applied. This was carried out in an assessment exercise by assuming a $20 \%$ increase in fuel price.

- Using only NOTs originating/destinating from/in a TAZ for crash prediction and consequently evaluating the safety impact of a TDM strategy will lead to a lack of some important information about the characteristics of reduced trips; i.e. NOTs, as an exposure variable, is not sensitive to trip time, trip length and route choice. For this reason, other exposure variables which are sensitive to the impact of trip assignment should also be taken into account. This was done by assigning traffic demand to the road network adopting the user equilibrium assignment method.

- The results of the comparison analysis revealed that the fuel-cost increase scenario has many impacts such as a reduction of total travel demand, total crash occurrence, VKT and VHT and mode shift. On the whole, there is a reduction of on average 105,485 daily trips (all types of modes) as a result of the fuel-cost increase scenario. This scenario also causes a reduction of 5.02 billion VKT per year, almost $11.57 \%$ of the total annual VKT in Flanders.

- The total NOCs is predicted to decrease by 2,133 for a period of 4 years. However, changes in the NOCs for different crash-type/severity-levels are not identical. As a result of an increase in the NOTs for the "Slowmode" category, crashes which involve vulnerable road users are predicted to increase; i.e. CSFS and CSSL crashes increased by $0.46 \%$ and $0.82 \%$ respectively. On the other hand, CCFS and CCSL crashes are predicted to decrease by $3.97 \%$ and $4.77 \%$ respectively. This reveals that the fuel-cost increase scenario affects different road users differently. The traffic safety situation slightly deteriorates for vulnerable road users; nevertheless, there are noticeable safety benefits for "Car-Car" crashes and for the overall traffic safety situation.

- When considering the changes in the NOCs at the TAZ level, it was found that the maximum reduction of "Car-Car" crashes and the maximum increase of "Car-Slowmode" crashes were both observed in urban areas (cities). It can be concluded that in cities, in contrast to other areas, there is a higher likelihood of finding an alternative mode for cars. In contrast, the TAZs in less urbanized regions and the TAZs nearby the borders usually lack good public transportation services. Therefore it is expected that we will not see many trips shift from cars to other modes in less urbanized areas and consequently there is a more stable traffic safety situation in these TAZs despite conducting the fuel-cost increase scenario.

This paper presents a new extension to the application of ZCPMs incorporated into TDM strategies. The results show the ability of ZCPMs as a reliable predictive tool which can be used during the planning level of transportation projects.

The fuel-cost increase scenario studied in this research investigated the relatively shortterm effects of an increased fuel price. In other words, the model is a short-term model in the sense that neither a shift in the composition of the vehicle fleet, nor changes in the location of businesses and/or the location choice for living as a result of the change in fuel cost are assumed.

Crashes are known to be a function of two components; exposure and risk. It is therefore likely that a fuel price increase will impact people's driving behavior and their speed choice; i.e. drivers might try to reduce their fuel consumption by driving more slowly. As a result, it can be 
assumed that the risk component will also decrease after the fuel-cost increase scenario implementation. In this study however, only the changes in the exposure component were taken into account, whereas the risk component was assumed to be constant. This might be a limitation of this study. If we were to include the risk component in this study as well, however, the traffic safety benefits might be expected to be even larger than predicted in this study.

Although some clear benefits (e.g. global traffic safety improvement or VKT reduction) are noticeable from the fuel-cost increase scenario, it would be beneficial to extend this study by including other TDM strategies in order to present a comprehensive traffic safety evaluation package.

In this study, as a first attempt, the methodology relied on the aggregate daily traffic information. Activity-based models are capable of providing disaggregate travel characteristics. Hence, different types of disaggregation based on time of day, day of the week, age, gender, motive, etc are in the list of potential future research in order to take full advantage of the output of activity-based models. 


\section{References}

Abdel-Aty, M., Siddiqui, C., Huang, H., 2011a. Integrating Trip and Roadway Characteristics in Managing Safety at Traffic Analysis Zones. In Transportation Research Board (TRB) 90th Annual Meeting, Washington D.C. USA.

Abdel-Aty, M., Siddiqui, C., Huang, H., 2011b. Zonal Level Safety Evaluation Incorporating Trip Generation Effects. In Transportation Research Board (TRB) 90th Annual Meeting, Washington D.C. USA.

Aguero-Valverde, J., Jovanis, P.P., 2006. Spatial analysis of fatal and injury crashes in Pennsylvania. Accident Analysis \& Prevention, 38(3), 618-625.

Amoros, E., Martin, J.L., Laumon, B., 2003. Comparison of road crashes incidence and severity between some French counties. Accident Analysis \& Prevention, 35(4), 537-547.

An, M., Casper, C., Wu, W., 2011. Using Travel Demand Model and Zonal Safety Planning Model for Safety Benefit Estimation in Project Evaluation. In Transportation Research Board (TRB) 90th Annual Meeting, Washington D.C. USA.

Arentze, T.A., Timmermans, H.J.P., 2004. ALBATROSS - Version 2.0 - A learning based transportationoriented simulation system. EIRASS (European Institute of Retailing and Services Studies).

Arentze, T.A., Timmermans, H.J.P., 2005. Incorporating Parametric Action Decision Trees in Computational Process Models of Activity-Travel Behavior: Theory and Illustration. Advanced OR and AI Methods in Transportation, 567-572.

Bellemans, T., Kochan, B., Janssens, D., Wets, G., Arentze, T.A., Timmermans, H.J.P., 2010. Implementation Framework and Development Trajectory of the FEATHERS Activity-Based Simulation Platform. Transportation Research Record, 2175, 111-119.

Chi, Guangqing, Cosby, A.G., Quddus, M.A., Levinson, D., Gilbert, P.A., 2010. Gasoline Prices and Traffic Safety in Mississippi. Journal of Safety Research, 41(6), 493-500.

Davidson, W., Donnelly, R., Vovsha, P., Freedman, J., Ruegg, S., Hicks, J., Castiglione, J., Picado, R., 2007. Synthesis of first practices and operational research approaches in activitybased travel demand modeling. Transportation Research Part A: Policy and Practice, 41(5), 464488.

Grabowski, D.C., Morrisey, M.A., 2004. Gasoline prices and motor vehicle fatalities. Journal of Policy Analysis and Management, 23(3), 575-593.

De Guevara, F.L., Washington, S.P., Oh, J., 2004. Forecasting crashes at the planning level. A simultaneous negative binomial crash model applied in Tucson, Arizona. Transportation Research Record, 1897, 191-199.

Goodwin, P., Dargay, J., Hanly, M., 2004. Elasticities of road traffic and fuel consumption with respect to price and income: a review. Transport Reviews, 24(3), 275-292.

Hadayeghi, A., 2009. Use of Advanced Techniques to Estimate Zonal Level Safety Planning Models and Examine Their Temporal Transferability. PhD thesis, Department of Civil Engineering, University of Toronto. 
Hadayeghi, A., Shalaby, A., Persaud, B., 2010. Development of Planning-Level Transportation Safety Models using Full Bayesian Semiparametric Additive Techniques. Journal of Transportation Safety \& Security, 2(1), 45-68.

Hadayeghi, A., Shalaby, A., Persaud, B., 2003. Macrolevel Accident Prediction Models for Evaluating Safety of Urban Transportation Systems. Transportation Research Record, 1840(1), 87-95.

Hadayeghi, A., Shalaby, A., Persaud, B., 2007. Safety Prediction Models: Proactive Tool for Safety Evaluation in Urban Transportation Planning Applications. Transportation Research Record, 2019(-1), 225-236.

Hadayeghi, A., Shalaby, A.S., Persaud, B.N., 2010. Development of planning level transportation safety tools using Geographically Weighted Poisson Regression. Accident; Analysis and Prevention, 42(2), 676-688.

Hadayeghi, A., Shalaby, A.S., Persaud, B.N., Cheung, C., 2006. Temporal transferability and updating of zonal level accident prediction models. Accident Analysis \& Prevention, 38(3), 579589.

Hintze, J. L., Nelson, R. D., 1998. Violin plots: A box plot-density trace synergism. American Statistician, 52 (2), 181-184.

Huang, H., Abdel-Aty, M., Darwiche, A., 2010. County-Level Crash Risk Analysis in Florida. Transportation Research Record, 2148(-1), 27-37.

Janssens, D., Wets, G., Timmermans, H.J.P., Arentze, T.A., 2007. Modelling Short-Term Dynamics in Activity-Travel Patterns: Conceptual Framework of the Feathers Model. In 11th World Conference on Transport Research, Berkeley CA, USA.

Kochan, B., Bellemans, T., Janssens, D., Wets, G., 2008. Assessing the Impact of Fuel Cost on Traffic Demand in Flanders Using Activity-Based Models. In Travel Demand Management TDM, Vienna, Austria.

Kochan, B., Bellemans, T., Janssens, D., Wets, G., 2012. Validation of an Activity-Based Traffic Demand Model for Flanders Implemented in the Feathers Simulation Platform. Book chapter in "Computational Intelligence for Traffic and Mobility", Atlantic Press, Forthcoming.

Levine, N., Kim, K.E., Nitz, L.H., 1995. Spatial analysis of Honolulu motor vehicle crashes: II. Zonal generators. Accident Analysis \& Prevention, 27(5), 675-685.

Litman, T., 2006. Mobility Management Traffic Safety Impacts. In Transportation Research Board (TRB) 85th Annual Meeting, Washington D.C. USA.

Litman, T., Fitzroy, S., 2011. Safe Travels: Evaluating Mobility Management Traffic Safety Impacts. Victoria Transport Policy Institute, www.vtpi.org (last accessed 17.03.2012).

Litman, T., 2010. Changing Vehicle Travel Price Sensitivities: The Rebounding Rebound Effect. Victoria Transport Policy Institute, www.vtpi.org (last accessed 17.03.2012).

Litman, T., 2011. Pricing For Traffic Safety: How Efficient Transport Pricing Can Reduce Roadway Crash Risk. Victoria Transport Policy Institute, www.vtpi.org (last accessed 17.03.2012). 
Lord, D., Mannering, F., 2010. The statistical analysis of crash-frequency data: A review and assessment of methodological alternatives. Transportation Research Part A: Policy and Practice, 44(5), 291-305.

Lovegrove, G.R., Litman, T., 2008. Using Macro-Level Collision Prediction Models to Evaluate the Road Safety Effects of Mobility Management Strategies: New Empirical Tools to Promote Sustainable Development. In Transportation Research Board (TRB) 87th Annual Meeting, Washington D.C. USA.

Lovegrove, G.R., Sayed, T., 2006. Macro-Level Collision Prediction Models for Evaluating Neighbourhood Traffic Safety. Canadian Journal of Civil Engineering, 33, 609-621.

Lovegrove, G.R., Sayed, T., 2007. Macrolevel Collision Prediction Models to Enhance Traditional Reactive Road Safety Improvement Programs. Transportation Research Record, 2019(-1), 65-73.

Naderan, A., Shahi, J., 2010. Aggregate crash prediction models: Introducing crash generation concept. Accident Analysis \& Prevention, 42(1), 339-346.

Noland, R.B., Oh, L., 2004. The effect of infrastructure and demographic change on trafficrelated fatalities and crashes: a case study of Illinois county-level data. Accident Analysis \& Prevention, 36(4), 525-532.

Noland, R.B., Quddus, M.A., 2004. A spatially disaggregate analysis of road casualties in England. Accident Analysis \& Prevention, 36(6), 973-984.

Pirdavani, A., Brijs, T., Bellemans, T., Kochan, B., Wets, G., (2012). Developing Zonal Crash Prediction Models with a Focus on Application of Different Exposure Measures. In: Transportation Research Board (TRB) 91st Annual Meeting, Washington D.C. USA.

Quddus, M.A., 2008. Modelling area-wide count outcomes with spatial correlation and heterogeneity: An analysis of London crash data. Accident Analysis \& Prevention, 40(4), 14861497.

Siddiqui, C., Abdel-Aty, M., Choi, K., Macroscopic spatial analysis of pedestrian and bicycle crashes. Accident Analysis \& Prevention, 45, 382-391.

Tarko, A.P., Inerowicz, M., Ramos, J., Li, W., 2008. Tool with Road-Level Crash Prediction for Transportation Safety Planning. Transportation Research Record, 2083(-1), 16-25.

Vovsha, P., Bradley, M., 2006. Advanced Activity-Based Models in Context of Planning Decisions. Transportation Research Record: Journal of the Transportation Research Board, 1981(-1), 34-41.

VTPI, 2011. Online TDM Encyclopedia, Victoria Transport Policy Institute. http://www.vtpi.org/tdm/index.php (last accessed 17.03.2012).

Wier, M., Weintraub, J., Humphreys, E.H., Seto, E., Bhatia, R., 2009. An area-level model of vehicle-pedestrian injury collisions with implications for land use and transportation planning. Accident Analysis \& Prevention, 41(1), 137-145. 